\title{
Thanks to Reviewers
}

\section{Phyllis Freeman ${ }^{1} \cdot$ Anthony Robbins ${ }^{1}$}

Published online: 25 March 2019

(c) Springer Nature Limited 2019

We appreciate this yearly opportunity to thank our reviewers. As this will be our last year as Editors of JPHP, we thank all of the reviewers with special emphasis! The single-most challenging task we have as journal editors is finding colleagues willing to review submissions and to complete the reviews.

Reviewers give us advice and help the authors of promising submissions to make them better. We received 413 submissions in the calendar year 2018 from at least 50 countries and sent 111 of them to the 85 outside reviewers listed below. Our authors regularly express their gratitude for the constructive guidance.

The countries include Afghanistan, Australia, Austria, Bahrain, Bangladesh, Canada, China, Congo (Democratic Republic Of The), Croatia, Denmark, Ecuador, Egypt, Ethiopia, France Germany Greece, Hong Kong, India, Indonesia, Iran, Ireland, Israel, Italy, Japan, Kazakhstan, Kenya, Korea (south, Republic Of), Macau, Malaysia, Mexico, Netherlands, Nigeria, Oman, Pakistan, Portugal, Romania, Saudi Arabia, Serbia and Montenegro, South Africa, Spain, Sweden, Switzerland, Taiwan, Thailand, Togo, Turkey, Uganda, United Kingdom, and the United States of America.

We thank all JPHP reviewers for the invaluable help. Several of those we list below provided more than one review; Roy Widdus completed 8-this year's record. Again, thanks to all.

Sachin R Atre

Jordan Alan Barab

Ruth Berkelman

Odilia Bermudez

Johannes Bircher

Eric C. Blank

Thomas Bodenheimer
Greet Cardon

Allison Chamberlain

James Chauvin

Yong Chen

Enrique Cifuentes

Bruce Cohen

Steven A. Cohen

Phyllis Freeman

phyllis.freeman@umb.edu

Anthony Robbins

anthony.robbins@tufts.edu

1 Boston, USA 
Ornella Corazza

Lisa Cosgrove

Jason E Davis

Richard A. Daynard

Kora DeBeck

Alfred DeMaria

Barbara Demeneix

Deborah V Edidin

Charles Farmer

David S. Fedson

John Frank

Elsie Freeman

Nicholas Furtado

Adam Gaffney

Norman Giesbrecht

Christopher Griggs

Jan Hendriks

Erin Hennessy

Louise Howard

Alessandro Iellamo

Dagmawi Iyasu

Sepideh Kaffash

Simeon Kimmel

Lydia Kline

Sheldon Krimsky

Agnes Kurniawan

Louis Michael Kyriakoude

Philip J. Landrigan

Christian Laurent

$\mathrm{Su}$ Yeon Lee-Tauler

Lynne Levitsky

Jane Lipscomb

Peter Grant Lurie

Gillian MacNaughton

Madhumita Dobe

Adolfo Martinez Palomo
Robert Morris

Subrata Mukherjee

Bob C. Mulder

Carles Muntaner

Elena N Naumova

Maya Negev

Celestino Obua

Julia Elisabeth Oettingen

Allison Patton

Lewis Pepper

Mark Pettigrew

Regina Rabinovich

Leon S. Robertson

Lance Rodewald

David Rosner

Alberto Salomone

Fred Schmidt

Robert Schweitzer

Jordi Serratosa

Xiao Shi

Julie P Smith

Tim Stockwell

Sabina Strano Rossi

Ahmad Hatim Sulaiman

Madjid Tavana

Michael Touchton

Leonardo Trasande

Mark A Tully

Adolfo Martinez Valle

Ed van Klink

Patrick Webb

Julia Wei Wu

Melodie Wenz-Gross

Roy Widdus

Catherine Woods

Publisher's Note Springer Nature remains neutral with regard to jurisdictional claims in published maps and institutional affiliations. 